\title{
ATTITUDE OF DENTAL PRACTITIONERS AND PARENTAL ACCEPTANCE IN USING ANIMAL-ASSISTED THERAPY (AAT) IN BEHA VIOUR MANAGEMENT OF PEDIATRIC PATIENTS
}

Dr.Harshita Shukla ${ }^{4}$, Dr.Sadanand Kulkarni ${ }^{1}$, Dr.Ashish Bhondey ${ }^{2}$, Dr.Sneha Khekade ${ }^{3}$, Dr.Durga Bhattad ${ }^{4}$, Dr.Nilesh P.Rojekar ${ }^{4}$

1-Professor \& Guide, 2- Reader, 3- Senior Lecturer, 2- Postgraduate Student, Department Of Pedodontics and Preventive Dentistry, Swargiya Dadasaheb Kalmegh Smruti Dental College and Hospital, Nagpur

\begin{abstract}
Aim: To evaluate the acceptance of Dentist, Parents and child to therapy pets, child's choice of pet and to analyse attitude of dentist towards animal-assisted therapy in the paediatric dental set-up as an aid to improve behaviour of the child during the dental treatment. Materials and methods: A descriptive cross-sectional survey was conducted among 60 children and their parents and 108 dentists. Chi-square test was used to determine the association of independent variables with each of the main outcome of interest.Results: The consent to the presence of pet was given by $56.67 \%$ parents $(\mathrm{p}=0.0359$, Significant) Children who chose dog as their preferred pet were $46.67 \%$ those who chose cat as their preferred pet were $25 \%$ ( $p=0.049$, Significant) Almost $76 \%$ Dentist agreed that AAT helps in behaviour management for Pediatric dentist ( $p=0.0001, S)$. Conclusion: Animal-Assisted Therapy (AAT) can prove to be a good behaviour management technique and can be a part of the exclusive Pediatric dental clinic.
\end{abstract}

KEYWORDS: Animal-assisted therapy, Pediatric Dentist

\section{INTRODUCTION}

Animals play an important part in the everyday lives of the majority of humans, including children. From a young age, children are introduced to animals through books and stories, cartoon characters, toys, as well as by having pets. It has recently been proposed that humans may have a natural affinity for animals that is evident very early in life. ${ }^{1}$
Even though the human-animal bond has existed for thousands of years, it is only recently that the mutual benefits of this relationship have started to be investigated empirically. Having a companion animal, or simply being around an animal, has been found to positively influence both children and adults in many ways: physiologically (Allen, Shykoff\&Izzo, 2001), mentally (Moretti et al., 2011), socially (McConnell, Brown, Shoda, Stayton, \& Martin, 2011), emotionally (Geist, 
2011), and developmentally. ${ }^{2}$

The history of animals being used for therapeutic reasons was first documented in the 1800s. The York Psychiatric Hospital in England kept companion animals on the grounds due to their calming effects on the patients. Purposeful, routine activity was achieved through the patient's maintenance of the companion animals. Animals used as therapeutic aids were introduced into the United States by the American Red Cross in 1942. Pawling Air Force Convalescent Hospital in Pawling, New York, began a program using animals when a recovering serviceman asked for a dog to keep him company during his long stay. Pet FacilitatedTherapy (PFT) has been associated with benefits ranging from physical and psychological to increased socialization in a variety of settings. ${ }^{3}$

The therapeutic use of animals can occur in three basic ways: (i) pets are used as companions for individuals who are either living independently in their own home or in assisted living facilities; (ii) pets are used in institutions where they help to stimulate and/or be companions to the residents; and (iii) animals visit institutions to help stimulate the residents' interest and provide a topic of conversation. $^{4}$

Animal Assisted Interventions (AAI) is defined by the International Association of Human-Animal Interaction Organizations (IAHAIO) as goaloriented interventions that intentionally include or incorporate animals in health, education and human service (e.g., social work) for the purpose of therapeutic gains in humans. According to the objectives of the interventions, they can be distinguished into Animal Assisted Therapy (AAT), Animal Assisted Education (AAE) and Animal Assisted Activities (AAA). ${ }^{5}$

AAT involves the interaction between a patient and a trained animal that has been specially educated for the therapy. The animals that conduct AAT are the therapy animals. It is a formal structured method whereby animals are used to aid in improving the well-being of a person suffering from psychological or physical illness or injury (Beck \&Katcher, 1984; Cusack \& Smith, 1984; Arkow, 1987). ${ }^{6}$

The first known animal therapy dog was Jingles introduced in 1969 to help a severely withdrawn child. $^{7}$ In January 1999, the Mater Children's Hospital in Brisbane, Queensland, Australia, became the first paediatric hospital in Australia to undertake a formal strictly controlled animalassisted therapy (AAT) programme, incorporating evaluation and research. ${ }^{6}$ In March 2015, Italy sanctioned an agreement between the government and regional authorities on the document 'National guidelines for AAI'. According to this agreement, an AAT requires a medical prescription. ${ }^{5}$

Animal-assisted therapy programmes are widely used and well established in hospitals in Europe, the United Kingdom and North America. ${ }^{6}$

Little research has been done to evaluate the beliefs of practitioners about the role that animals can play in human health. Since practitioners are gatekeepers to treatments, understanding their beliefs about animals' utility in human health interventions is fundamental to the mainstream acceptance of animals to achieve specific therapy goals. ${ }^{8}$

\section{MATERIALS AND METHODS}

A descriptive cross-sectional survey was conducted among 60 children and their parents aged 3 to 12 years and their parents randomly selected from the Department of Paediatric and Preventive Dentistry and 108 practicing Dentist. Questionnaire was filled to assess the acceptance of parents and the choice of pet by the children in a dental clinic. The parents of the children were informed about the nature of the study. The age of the child was ascertained from the date of birth. Approximately, 20 children from each age group, that is 3-6 year (36-71 months), 6-9 year (72 to 107 months), and 9-12 year (108 to 144 months) were included in the study. 
Parents of children aged 3-12 years of age of all socio-economic status irrespective of their age, gender, income, educational status, and occupation who were willing to participate and gave consent.

Children with any Special health care needs were excluded from the study.

Parents and children were interviewed in the local language by the investigator. Power point image was shown to child, of most liked pets in the form of jpeg image. Patient was asked to select the favourite pet after seeing the image. The order of the pets shown was Dog, Cat, Rabbit, Fish, Tortoise, and Parrot.

A Questionnaire was distributed among 108 dentists. The questionnaire was developed based on the study objectives, taking guidance from previous literature. The study tool consisted of two sections. The first part consisted of three questions on the respondents' personal and professional background, such as age, gender, qualification. Second part consisted of 14 closed ended questions on dentists' attitudes/ opinions on AAT as an aid in behaviour management of pediatric patients.

Chi-square test was used to determine the association of independent variables with each of the main outcome of interest. $\mathrm{P}<0.05$ was considered to be statistically significant.

\section{STATISTICAL ANALYSIS}

Statistical significance was set at $5 \%$ level of significance $(p<0.05)$. Chi-square test was used to determine association of each of the independent variables (gender and qualification) with each of the main outcome of interest.

\section{RESULTS}

\section{PARENT AND CHILD'S ACCEPTANCE FOR AAT}

The total sample comprised of 60 children aged 3 to 12 years divided in three groups, 3-6 year (36-71 months), 6-9 year (72 to 107 months), and 9-12 year
(108 to 144 months). Of the 60 children, 34(56.6\%) were males and 26(43.3\%) were females and were accompanied along their parents.

Of the total 60 parents 34 consented to allow an animal pet of which 15 were parents of 9-12 year old , 12 were parents of 6-9 year old, and 7 were parents of 3-6 year old children. It was noticed that as the age increases there was more likelihood of parents giving consent for pet in the operatory which was statistically significant $(p=0.0359, S)$

Majority of the males (36.7\%) chose dogs and females chose cat $(16.67 \%)$ as the therapy pets.

Of the 60 children, the order of preference of the animal as therapy pet was dog $(46.7 \%)$, followed by cat $(25 \%)$, fish $(11.67 \%)$, rabbit $(6.67 \%)$, tortoise $(6.67 \%)$ and parrot $(3.33 \%)$.

The younger age group children i.e 3-6 years (20\%) preferred dog as the therapy pet followed by $9-10$ years old which was not significant statistically.

In the present study, number of children who opted for live pets $(73.33 \%)$ were more as compared to soft toys $(26.67 \%)$.

$53 \%$ of children desired the pet to be provided by clinic $(53.33 \%)$ compared to children who wanted their own pet $(46.67 \%)$ in the dental operatory.

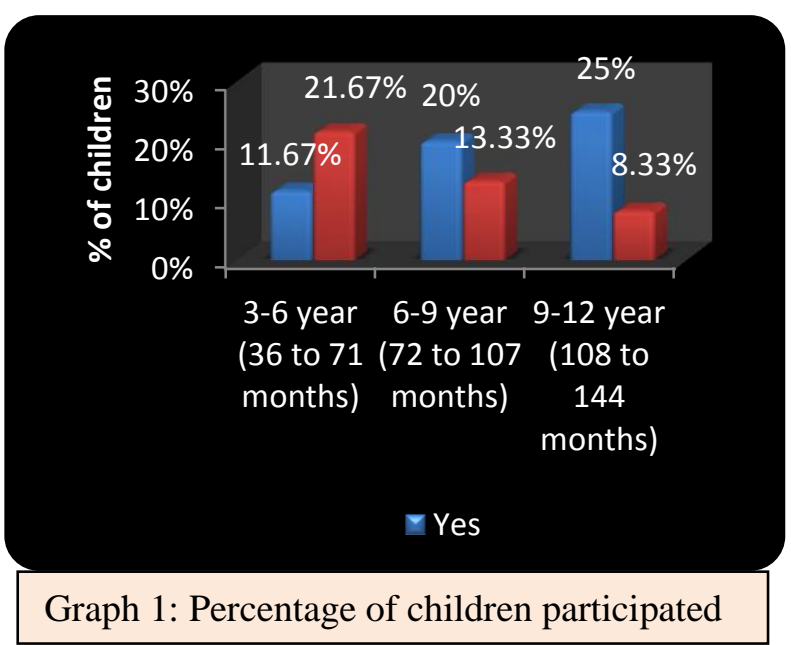




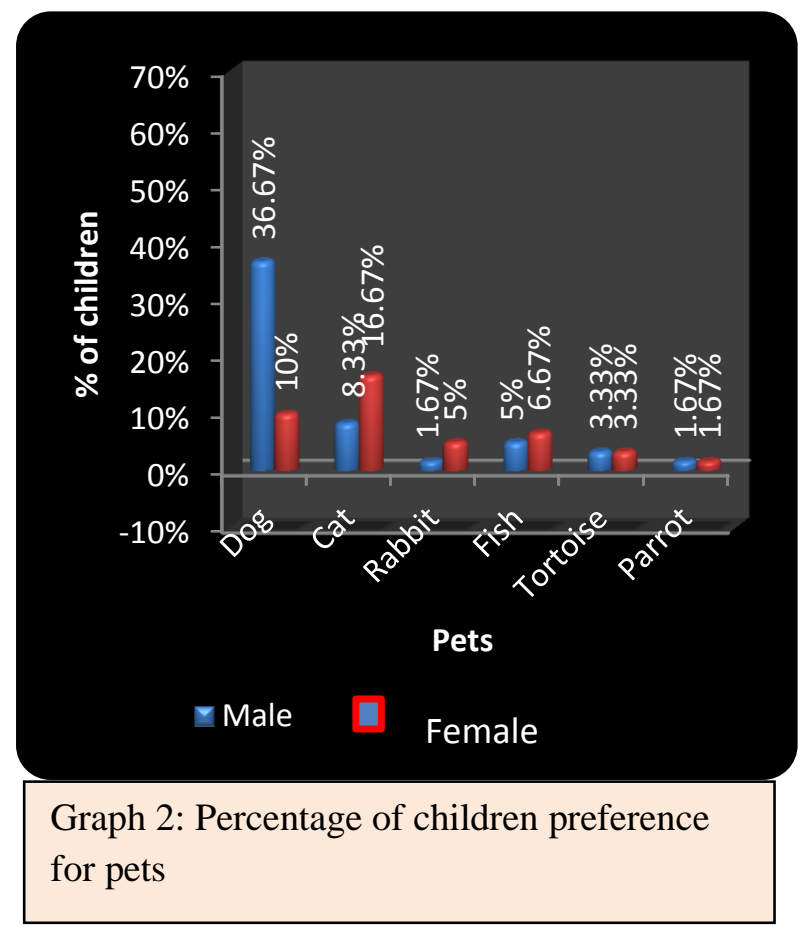

\section{DENTIST'S ACCEPTANCE FOR AAT}

Of total 108 dentists that participated in the study 51\%had an idea about AAT (Animal Assisted Therapy) which was found to be statistically significant. $55 \%$ of the dentist said that they were scared of animals. 59\% dentist agreed in implementing AAT at their clinic for pediatric patients and $70 \%$ agreed that AAT may help in behaviour management. $87 \%$ of dentist in the study had never practiced AAT at their clinic as $75 \%$ of dentists feel that patients maybe afraid / disturbed by animals, or animals in dental clinic will be disturbing to dentist while working. $87 \%$ of the dentist think hygiene problems may occur because of AAT, 94\% dentist feel having animals in clinic will increase maintenance issues and $85 \%$ of them think AAT may result in allergies from animals, scratches or bites. $22 \%$ dentist accepted that their cultural belief do not allow them to have animals in their clinic. Whereas $42 \%$ of the dentist feels having animals in clinic can result in loss of patient. All these results were found to be statistically significant $(\mathrm{p}=0.0001)$ although no significant differences were seen based on qualification of dentist.

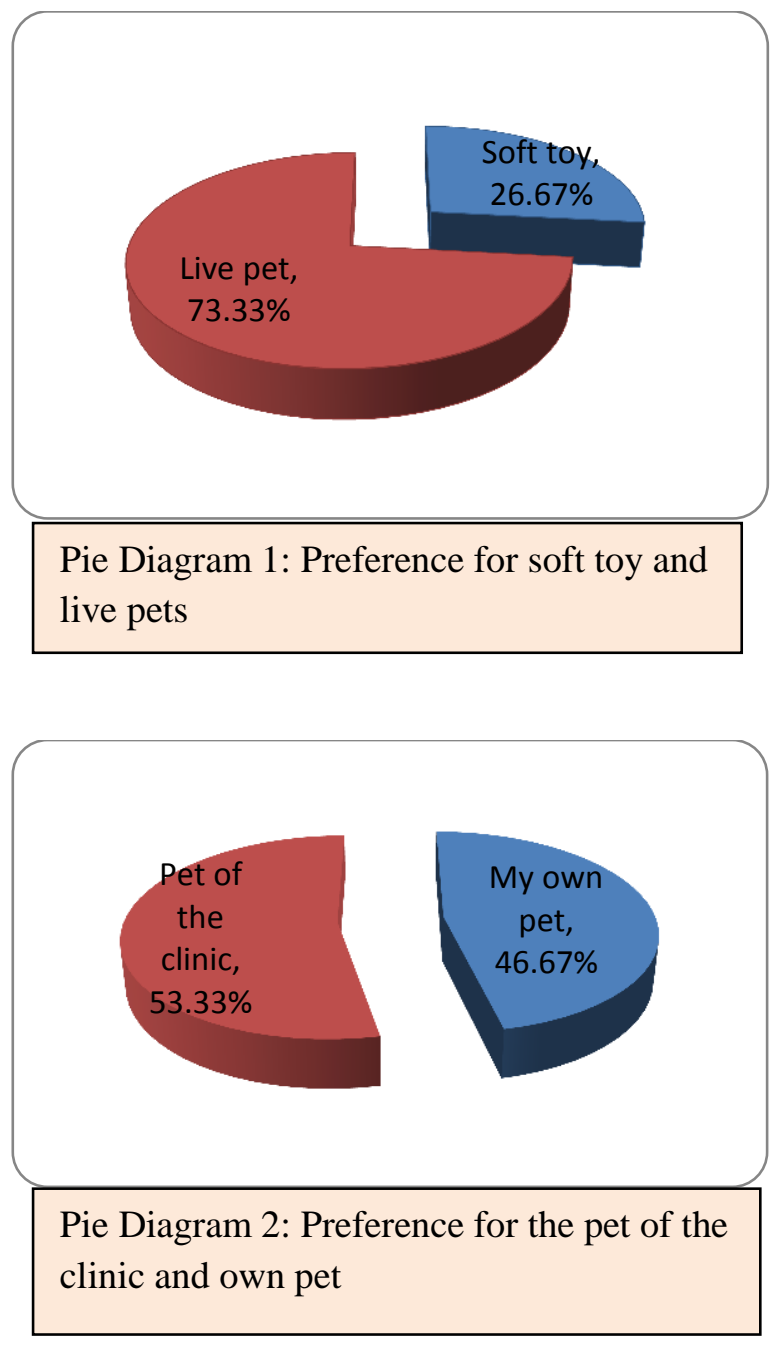

\section{DISCUSSION}

The first objective was to assess the parental acceptance to the presence of therapeutic pets in the paediatric dental set-up. In the study, mostly parents of the older children i.e 9-12 years consent for the presence of pet, whereas the parents of children age 3-6 years refused. This could be due to faith that animals and pets can spread infections to humans, especially kids. ${ }^{12}$ Thus, as the age increases there is more likelihood of parents giving consent for pet in the operatory.

Dog was the favourite pet of children. This could be because as it is the most common pet in the Indian households. Gupta et al (2018) stated dog was the favourite pet of children. ${ }^{7}$ Animals most notably dogs by virtue of their unique ability to offer nonjudgmental and unconditional positive regard even 
to unfamiliar persons, may be able to provide certain components of social support within the context of AAT. According to Levinson, dogs help protect some young children from environmental dangers. ${ }^{9}$

There was a statistically significant difference seen with gender and choice of pet $(\mathrm{p}=0.049, \mathrm{~S})$ with a majority of boys $22(36.67 \%)$ having dog as their choice, while girls $10(16.67 \%)$ preferred cats. Kidd (1987), no significant gender differences in the percentages attached to dogs or cats. ${ }^{1}$ Cat was a preferred pet for younger children in our studywhich could be because kittens being fluffy feline are childhood favourites according to American Academy of Pediatrics.

It is seen that moving objects catches infants' attention, but this attention stays only if it is reciprocal in nature because live pets could actually respond and interact with the babies, while the mechanical pets could be only moved towards the infants. This forms the basis of attachment in parent-child relationships, the same may be true in child-pet relationships. ${ }^{7}$

Dentist's attitude toward animal-assisted therapy (AAT) is crucial to the success of animal-assisted programs. Such programs are used with increasing frequency in the healthcare sector, especially within rehabilitation programs for patients with a broad range of problems. To maximize the success of these programs, it is important to understand dentist's attitudes toward animal-assisted therapy (AAT). ${ }^{10}$

In the present study $51 \%$ of dentist had an idea about AAT (Animal Assisted Therapy) which was found to be statistically significant.

It is important to consider the relationships with animals of involved staff and to respect these. For AAT to be successful, the involved dentists should have a good relationship with animals. $55 \%$ of the dentist stated they are scared of animals and majority of which were afraid from dog.
AAT is an applied science using animals to solve a human problem. It is an interdisciplinary approach using animals as an adjunct to other therapies. The use of animals has been a practice for a long time in hospitals such as one reporting the use of a golden retriever for $>6$ months in a hospital bringing smiles to the faces of patients and creation of a pet therapy program in the hospital itself. But its use in dentistry is still in its offsprings. In the present study, 64(59\%) dentist agreed with implementing AAT in clinic for pediatric patients but $94(87 \%)$ of dentist in the study had never practiced AAT in their clinic. ${ }^{11}$

It is possible that one of the barriers to implementation of AAT is the perceived beliefs of others or the culture of the institutions that practitioners work within. Evidence of cultural or regional differences in implementation of AAT were hinted at by Rice et al. (1973) who described geographic clustering of AAT use. 24(22\%) dentist in the current study accepted that their cultural belief do not allow them to have animals in their clinic. $^{8}$

$75 \%$ of dentist felt that patients may be afraid / disturbed by animals and $77 \%$ felt that presence of animals in dental clinic while working maybe disturbing to dentist.

$85 \%$ of them think AAT may result in allergies from animals, scratches or bites. Since the early 1990s, there has been much research on the effect of animal exposure on the development of allergies and/or asthma.

94\% dentist feel having animals in clinic will increase maintenance issues, Several studies have found that the majority of therapists utilizing animals in therapy are self-taught on the subject, lacking formal education or training in AAT. However, the lack of formal training is not due to a lack of interest in training programs. In fact many mental health practitioners report desiring formal training or education in AAT. The discrepancy between practitioners wanting training but not 
receiving it demonstrates the need for better education and training mechanisms for professionals who wish to engage in AAT. ${ }^{8}$

Healthcare facilities that want to introduce animals often face the problem of fears regarding hygiene and patient security. $87 \%$ of the dentist in this study think hygiene problems may occur because of $\mathrm{AAT}^{10}$ while $42 \%$ of the dentist felt that presence of animals in clinic can result in loss of patient.

$70 \%$ agreed that AAT helps in behaviour management for paediatric dentist as it is a holistic method.

While conducting an AAT, Barker et al. (2005) observed that the staff members, would voluntarily play with the therapy dogs that came to visit the patients which led them to study the effect that interaction with the therapy dog could have on staff members' stress levels. They concluded that spending as little as 5 minutes with a therapy dog proved to be effective at lowering staff members' cortisol levels. $^{8}$

\section{CONCLUSION}

AAT can prove to be a good behaviour management technique and a great addition to practice since a key to a good pediatric practice is reduction of anxiety and controlling behaviour. Animal assisted interventions can create a more comfortable environment for both patients and dentist. Appropriate patient consent should be taken before practicing AAT in clinic. The pets should be properly selected, immunised on time to reduce chances of allergies and properly trained in animal training institutes. Since pet therapy is in its infancy, in India, further studies need to be done.

Financial support and sponsorship: Nil.

Conflicts of interest: There are no conflicts of interest.

\section{REFERENCES}

1. Lobue V, Pickard MB, Sherman K, Axford C, Deloache JS. Young children's interest in live animals. 2013;57-69.

2. Noga Lutzky-CohenDepartmentMotivation to use animal-assisted therapy (AAT) among counsellingand clinical psychology graduate students 2016.

3. Marie H. Therapists Using Pet-Facilitated Therapy. 1996;10-7.

4. Velde BP, Cipriani J, Fisher G. Resident and therapist views of animal-assisted therapy: Implications for occupational therapy practice. 2005;43-50.

5. Pinto A, Santis M De, Moretti C, Farina L, Ravarotto L. Complementary Therapies in Medicine Medical practitioners' attitudes towards animal assisted interventions . An Italian survey. Complement Ther Med [Internet]. Elsevier; 2017;33:20-6.

6. Mbbs WJM. Attitudes of paediatric medical ward staff to a dog visitation programme. 2002;537-44.

7. Gupta N, Yadav T. Parent's acceptance and their children's choice of pet for animal-assisted therapy (A.A.T.) in 3- to 12-year-old children in the dental operatory - A questionnaire-based pilot study. 2018;1-7.

8. Trembath F. Practitioner Attitudes and Beliefs Regarding the Role Animals Play in Human Health. 2014;1-7.

9. Kruger KA. Can Animals Help Humans Heal? Animal-Assisted Interventions in Adolescent Mental Health. June 2004.

10. Hediger K, Hund-georgiadis M. Animalassisted therapy in the view of staff members before and after implementation in a rehabilitation clinic 2017. 
11. Nammalwar RB, Rangeeth P. Original Article A bite out of anxiety: Evaluation of animal assisted activity on anxiety in children attending a pediatric dental outpatient unit. 2018;181-4.

12. Kids health from Nemours for parents. https://kidshealth.org/en/parents/pet-infections.html 